\title{
A Team-Based E-Learning Method for Clinical Education in the COVID-19 Pandemic.
}

\author{
Sedighe Sadat Hashemikamangar (iD ${ }^{1}$ and Mehrzad Gholampourdehaki (iD) ${ }^{2,{ }^{*}}$ \\ ${ }^{1}$ Operative Department, Dental School, Tehran University of medical sciences, Tehran, Iran \\ ${ }^{2}$ Internal Medicine Department, AJA University Medical Sciences, Tehran, Iran \\ "Corresponding author: Internal Medicine Department, AJA University Medical Sciences, Tehran, Iran. Email: mehr.ghol@gmail.com \\ Received 2021 May 17; Revised 2021 June 28; Accepted 2021 July 01
}

\begin{abstract}
Background: Following the emergence of the new coronavirus pandemic, e-learning seemed to be the first and best alternative to classroom-based medical education. The Learning Management System (LMS) is a key infrastructure requirement for the provision and progression of e-learning.

Objectives: This brief report is about a team-based e-learning method for clinical education in the COVID-19 pandemic.

Methods: A novel e-learning protocol was implemented for the instruction of knowledge-based part of practical operative dentistry course to third- and fourth-year dental students in NAVID LMS, and their satisfaction levels were evaluated.

Results: More than $90 \%$ of the students mentioned that the method encouraged them to learn new topics and created a joyful learning experience for them. More than $80 \%$ reported that the method decreased their anxiety for learning practical topics during the COVID-19 pandemic.

Conclusions: Implementation of such a team-based e-learning method can greatly help in the instruction of clinical topics.
\end{abstract}

Keywords: Medical Education, Team_Based Learning, E_Learning

\section{Background}

The coronavirus disease 2019 (COVID-19) pandemic had over 181 million confirmed cases and more than 3900000 deaths by July 26, 2021 (https://coronavirus.jhu.edu/map.html). Billions of people all over the world practiced quarantine, isolation, or social distancing to contain the outbreak and prevent healthcare collapse. Due to close contact between the patient and dental clinician, both dental patients and dental clinicians are at high risk of infection with the novel coronavirus during dental procedures (1). Thus, many dental schools worldwide were closed. Iranian dental schools were no exception to this rule, and conventional classroom-based dental education was temporarily replaced by e-learning.

E-learning is currently a well-established educational modality for medical education, and its application for dental education is also on the rise (2). Scientific evidence on the efficacy of dental curricula confirms the optimal efficacy of e-learning as well as blended learning in different fields (3). Thus, following the emergence of the new coronavirus pandemic, e-learning seemed to be the first and best alternative to classroom-based dental education for theoretical modules.
Team-based learning (TBL) is an active learning strategy that is learner-centered. It is made up of a sequence of activities designed to allow the instructor to provide frequent feedback to students. The structure and activities in TBL are designed to hold students accountable for collaboration with peers. TBL moves beyond the basic acquisition of facts by emphasizing the importance of applying knowledge to real-life scenarios through intragroup and intergroup discussions of problems that are designed to foster complex reasoning and debate (4). In the past decade, there has been a move to blend information technology (IT) with active learning strategies, like TBL (4).

Evidence shows that interactions and communications between the learners are imperative for optimal efficacy of e-learning, and would create a joyful learning experience for them (5). Thus, a TBL was designed for this purpose.

\section{Objectives}

This study was done to introduce and evaluate a novel team-based e-learning method for clinical education in the COVID-19 pandemic. 


\section{Methods}

The School of Dentistry of Tehran University of Medical Sciences (TUMS) started virtual instruction of all theoretical courses of the undergraduate dental curriculum immediately after the temporary closure of the university due to the COVID-19 pandemic. They had employed NAVID, a novel university learning management system (LMS), accessible at http://tumsnavid.vums.ac.ir/ for e-learning. The LMS is a key infrastructure requirement for the provision and progression of e-learning. The LMS performs a wide range of tasks, including application, subscription, management, follow-up, scientific assessment, program provision, interlearner interactions, presentation of educational contents, and learner-mentor communication. NAVID is a national LMS exclusive to the Iranian universities of medical sciences and is currently used by over 70 academic centers in Iran. This electronic online LMS has the following features: (1) Introducing the topic to be taught, which is the first route of communication between the mentor and students. (2) Different sections related to the topic in the LMS include:

-Contents, resources, and references: In this menu, the educational content and the related references are present.

-Homework: The homework of students is uploaded in this section.

-Tests: This system is also capable of designing tests to allow self-assessment by students.

-Chat: A chat room or forum, which enables the communication between students asynchronously.

-Classes: Synchronous and online classes are held in this menu.

-Messages: This feature allows sending and receiving messages between the mentors and student(s), and also between students.

-Reports: This feature discloses the rate of participation of students.

The features of the LMS are suitable for the instruction of theoretical dental courses. Dental curricula in dental schools of Iran, such as the School of Dentistry of TUMS include clinical hands-on courses after preclinical courses. Considering the long-term closure of universities and cancellation of all clinical instructions during the COVID-19 pandemic, there would probably be a time shortage for the instruction of all the clinical topics after the reopening of universities, and there would be no other way than compacting the clinical courses. Thus, the authorities decided to design a theoretical module for clinical courses to teach the related topics to students using the NAVID LMS.

This novel e-learning protocol was implemented for the instruction of knowledge-based part of practical op- erative dentistry course to third- and fourth-year dental students of the International campus of Dental School in April 2020 (for practical courses, the students were divided into small groups in NAVID LMS; practical operative dentistry courses were instructed to students in 10 groups of 5 students each). (1) A seminar topic with practical content related to the clinical requirements of the course was selected by the mentor for each group. (2) Students in each group selected a team leader and introduced him/her to the mentor via a message in the NAVID LMS. The team leader was responsible for sharing responsibilities, coordination between the group members, and communication with the mentor. (3) The seminar topic was loaded by the mentor as homework for the respective group. (4) The students in each group had to work on the seminar as teamwork and prepare it within two weeks by sharing responsibilities between the members. For this purpose, they had to search the scientific databases to provide content, write the seminar paper along with images showing the procedural steps, prepare a PowerPoint presentation along with the images of clinical steps with a maximum of 10 slides, record a narration during the PowerPoint presentation, produce a small multimedia file, design 5 fourchoice questions from the seminar content, and search the Internet and find a short film relevant to the seminar topic. (5) The seminar paper, the multimedia file, and the film were sent to the mentor by the team leader in response to the homework. (6) The mentor performed the necessary corrections/modifications and uploaded the seminar paper, film, and multimedia in the "contents and resources" menu of the system for use by all students of the group. The questions related to the seminar were uploaded in the test menu for self-assessment. (7) After one week, a forum was activated in NAVID LMS for each seminar topic, so that the students could interact and brainstorm with the mentor regarding the challenges of the seminar topics and clinical implications.

Finally, the students were provided with a questionnaire to assess their level of satisfaction with the program. The questionnaire included the following three questions: (1) Did this technique of learning encourage you to learn new topics? (2) Did this method of learning decrease your anxiety for learning practical topics during the COVID-19 pandemic? (3) Did this method of learning create a joyful learning experience for you?

\section{Results}

The followings are the satisfaction results of 50 students: (1) Did this technique of learning encourage you to learn new topics? Of all, $92 \%$ gave a positive, and $8 \%$ gave a negative response to this question. (2) Did this method of 
learning decrease your anxiety for learning practical topics during the COVID-19 pandemic? Of all, $84 \%$ gave a positive, and $16 \%$ gave a negative response to this question. (3) Did this method of learning create a joyful learning experience for you? Of all, $94 \%$ gave a positive, and $6 \%$ gave a negative response to this question.

\section{Discussion}

Considering the COVID-19 pandemic and subsequent closure of dental schools and anxiety of dental students regarding their education, particularly in clinical topics, it seems that the implementation of such techniques using the existing LMS features can greatly help in the instruction of dental clinical topics. Based on the positive answers to questions 2 and 3, it seems that using TBL in a virtual learning environment can provide joyful learning of clinical medical education.

\section{Footnotes}

Authors' Contribution: Study concept and design: Both authors; Acquisition of data: Both authors; Analysis and interpretation of data: Both authors; Drafting of the manuscript: both authors; Critical revision of the manuscript for important intellectual content: Both authors; Statistical analysis: Second author; Administrative, technical, and material support: First author; Study supervision: Both authors.

Conflict of Interests: The authors confirm that there is no conflict of interest to declare.

Ethical Approval: This was a novel method of education not a study, and does not need ethic code.

Funding/Support: None.

\section{References}

1. da Cruz Perez DE, Passos KKM, Machado RA, Martelli-Junior H, Bonan PRF. Continuing education in oral cancer during coronavirus disease 2019 (covid-19) outbreak. Oral Oncol. 2020;105:104713. doi: 10.1016/j.oraloncology.2020.104713. [PubMed: 32307326]. [PubMed Central: PMC7162637].

2. Gormley GJ, Collins K, Boohan M, Bickle IC, Stevenson M. Is there a place for e-learning in clinical skills? A survey of undergraduate medical students' experiences and attitudes. Med Teach. 2009;31(1):e6-12. doi: 10.1080/01421590802334317. [PubMed: 19253150].

3. Hashemikamangar SS, Yazdanpanah F, Mirzaii M, Yazdani R, Karazifard MJ, Yasini E. Efficacy of e-learning via the website of Tehran University of Medical Sciences for diagnosing tooth discolorations and treatment planning by senior dental students. Acta Med Iran. 2016;54(8):536-41. [PubMed: 27701725].

4. Koh YYJ, Schmidt HG, Low-Beer N, Rotgans JI. Team-based learning analytics: An empirical case study. Acad Med. 2020;95(6):872-8. doi: 10.1097/ACM.0000000000003157. [PubMed: 31972678]. [PubMed Central: PMC7242170].

5. Roberts BS, Roberts EP, Reynolds S, Stein AF. Dental students' use of student-managed google docs and other technologies in collaborative learning. J Dent Educ. 2019;83(4):437-44. doi: 10.21815/JDE.019.053. [PubMed: 30745349]. 